\title{
Piezo-Micro-Ultrasound-Transducers for Air-Coupled Arrays: Modelling and Experiments in the Linear and Non-Linear Regimes
}

\author{
Gianluca Massimino ${ }^{\mathrm{a}}$, Alessandro Colombo ${ }^{\mathrm{a}}$, Raffaele Ardito ${ }^{\mathrm{a}}$, Fabio \\ Quaglia $^{\mathrm{b}}$, Alberto Corigliano ${ }^{\mathrm{a}, *}$ \\ ${ }^{a}$ Department of Civil and Environmental Engineering, Politecnico di Milano, Piazza \\ Leonardo da Vinci, 32, 20133 Milan, Italy \\ ${ }^{b}$ Analog, MEMS \& Sensors Group, ST Microelectronics, Via Tolomeo 1, 20010 Cornaredo, \\ Italy
}

\begin{abstract}
The paper is focused on the multi-physics modelling, via the finite element method (FEM), of a 4x4 air-coupled array of Piezoelectric Micromachined Ultrasonic Transducers (PMUTs), and on its mechanical and acoustic validation by means of the comparison, in the time domain, with experimental results.

A two-stage numerical procedure is employed, in order to evaluate the complete performance of the device, by means of two FEM models. In the first stage, the electro-mechanical-acoustic (EMA) problem is solved for the standalone transducer, taking into account the fabrication induced residual stresses and the multiple couplings between different physics. The numerical results are compared with the experimental ones in terms of initial deflection and time histories of pressure, in the linear and non-linear regime: the proposed model correctly captures the reported phenomena and perfectly matches the experimental trends.

The second stage is devoted to the simulation of the $4 \times 4$ PMUTs array performance, belonging to the silicon die. The vibrating plates are modelled as equivalent rigid pistons. The acceleration histories, computed in the first stage, are imposed on the pistons, while a rigid baffle condition is enforced on the
\end{abstract}

\footnotetext{
* Corresponding author

Email address: alberto.corigliano@polimi.it (Alberto Corigliano)
} 
remaining part of the die surface. The model is adopted to predict the pressure field for different patterns of the array's activation.

Keywords: acoustic-structure interaction, array, multiphysics modelling, non-linear dynamics, Piezoelectric Micromachined Ultrasonic Tranducers (PMUTs), ultrasound

\section{Introduction}

Piezoelectric Micromachined Ultrasonic Transducers (PMUTs) are layered diaphragms with a piezoelectric layer for emitting and receiving ultrasonic waves [1, 2]. Nowadays, they are employed in several applications: in-air propagation is

5 exploited for gesture recognition and range-finding [3, 4, 5]; in-water propagation is used for micropumps [6, 7], sonography [ 8 and finger-printing recognition [9].

This work is focused on an air-coupled array of $4 \times 4$ circular transducers (Fig. 1) with performing frequency of $100 \mathrm{kHz}$ [10]. A piezoelectric thin film, made of lead zirconate titanate (PZT) [1, 12] is deposited by the sol-gel technique in a coaxial hat configuration on the structural silicon diaphragm. Several examples of PMUTs operating in air are presented in the literature, with typical performing frequency above $200 \mathrm{kHz}[13$. The use of lower operating frequencies is associated with wider plates and, as a consequence, a paramount role is played by the geometric non-linearities, related to the very high aspect ratio ${ }_{15}$ (diameter/thickness) of the considered transducers.

The goal of this work is to simulate the non-linear dynamic oscillation due to the involved large displacements of the system [14, 15, that generates the pressure waves into the surrounding air [16. The non-linear behaviour is associated with a threshold of the applied voltage, above which the vibration amplitude increases very slowly with respect to the increments of the input voltage. The simulation and experimental validation in the non-linear regime is an important difference with respect to previously published works. As an example, in [1] and [17] only the linearized small signal frequency response of the coupled electromechanical-acoustic system is considered; hence, there is no reference to 


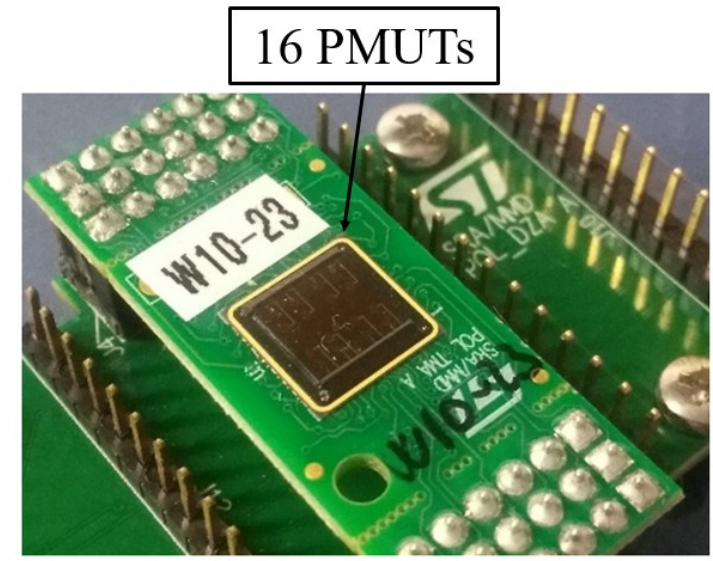

Figure 1: View of $4 \times 4$ array of circular PMUTs.

the non-linear dynamic behaviour due to the involved large displacements.

A two stage numerical technique is employed in order to evaluate the complete performance of the system, based on the diaphragms response, by means of two FEM models.

In the first stage, the 2D fully coupled electro-mechanical-acoustic (EMA) 30 problem is solved considering the stand-alone transducer in the transmitting (TX) phase, exploiting the axial symmetry. This FEM model provides the transducer acceleration time history due to the imposed voltage perturbation.

The $4 \times 4$ array response is computed in a second step, in which an equivalent rigid piston is considered instead of the PMUT diaphragm. The imposed vertical 35 accelerations are extracted from the analyses carried out with the initial model. Moreover, a hard wall condition is enforced on the silicon die surface in order to simulate a rigid baffle.

As a matter of fact, the latter model is devoted to the 3D acoustic simulation of the full set of PMUTs. This approach represents a useful tool to study beam-forming problems with reduced computational cost, with respect to the time-consuming fully coupled electro-mechanical-acoustic FEM model.

In order to evaluate the influence of the static voltage on the initial deflected configuration and the fundamental frequency, an experimental campaign 
has been carried out by means of the Polytec MSA-500 interferometer and vibrometer. Additionally, the comparison between the experimental and numerical hard/soft spring frequency response functions (FRFs) curve of the system is presented. The measurements are obtained by means of a set of frequency sweeps at different constant bias voltages and voltage amplitudes. Then, the steady state vertical displacement amplitudes at the center of the PMUT has been collected for each input frequency, bias level and input voltage amplitude.

The paper is organized as follows. In Section 2, the fully coupled EMA numerical model for the clamped circular single transducer is described in the 2D axisymmetric meridian half plane, along with the $4 \mathrm{x} 4$ array of PMUTs 3D acoustic model. In Section 3, the experimental tests and the measurements are presented in order to validate the numerical model for the single diaphragm, in the mechanical and in the acoustic domains. Moreover, the numerical predictions in the case of the TX phase for the PMUTs array are presented and compared with the single transducer ones. The last section is devoted to the closing remarks.

\section{Numerical modelling for the single transducer}

In this section the two FEM models, built in COMSOL Multiphysics 5.4 are presented. The former is referred to a single transducer, so that the axial symmetry of the system is considered. Therefore, the EMA problem is solved in the meridian half space, modelling the diaphragm and the air cavity. The PMUT has a radius of $440 \mu \mathrm{m}$ and an overall thickness equal to $8 \mu \mathrm{m}$, such that the diameter/thickness aspect ratio is 110. The thickest structural layer is made of silicon with thickness equal to $4.25 \mu \mathrm{m}$. The PZT layer has thickness equal to $1.06 \mu \mathrm{m}$ and it is in a circular hat configuration with radius of 308 $\mu \mathrm{m}$, coaxial with the structural diaphragm. The transducer is clamped over an air-filled closed cylindrical cavity with height equal to $400 \mu \mathrm{m}$ and the same radius of the upper diaphragm.

The FEM model, shown in Fig. 2, is characterized by the following physics: 
elastodynamics in the linear elastic layered system, electrostatics in the piezoelectric layer with the enforced linear stress-charge law and linear pressure acoustics in the air cavity and in the half semi-circular air domain with radius equal to $2 \lambda$, where $\lambda=c / f_{0}=3434 \mu \mathrm{m}$ is the in-air wavelength at the performing frequency $f_{0}=100 \mathrm{kHz}, c=343 \mathrm{~m} / \mathrm{s}$ is the in-air speed of sound at the thermodynamic reference state of $T_{r e f}=293.15 \mathrm{~K}$ and $P_{r e f}=1 \mathrm{~atm}$. Furthermore, the calculated Rayleigh distance, denoting the transition between the near-field domain and the far-field domain is equal to $D_{R}=S / \lambda=177.1 \mu \mathrm{m}$, where $S$ is the PMUT area [18.

The layered diaphragm is subjected to a certain amount of initial stress due to the micromachining fabrication process. Therefore, the residual stress state is taken into account by an amount of initial stress isotropically distributed in the plane of each layer. As a matter of fact, both tensile and compressive stresses are present in the PMUT cross section. The described pre-stress state has been estimated by means of a set of static measurements, on reference structures belonging to the silicon wafer, together with suitable finite element simulations. Therefore, the procedure allows for the identification of the stress level in each layer. The FE mesh consists of linear quadrilateral elements with maximum element size equal to $0.5 \mu \mathrm{m}$. Additionally, each layer is discretized using 1 element but for the PZT and the structural silicon layers, in which 2 and 10 finite elements through the thickness are employed, respectively. The acoustic computational domain consists of triangular linear elements with a maximum size equal to $\lambda / 6=572.33 \mu \mathrm{m}$. Therefore, the fully coupled electromechanicalacoustic model is characterized by a total number of degrees of freedom equal to 74690 .

A proper damping is enforced in the elastodynamics equations by means of the Rayleigh formulation, in order to simulate the various energy losses such as thermo-elastic losses, surface and interface dissipation, anchor losses. The acoustic medium is considered non-dissipative, thus neglecting the thermo-viscous properties of air [19, 20]. The computed Rayleigh damping parameters refer to $\xi=1 /\left(2 Q_{\text {struct }}\right)$ where $Q_{\text {struct }}$ is the imposed structural quality factor in 


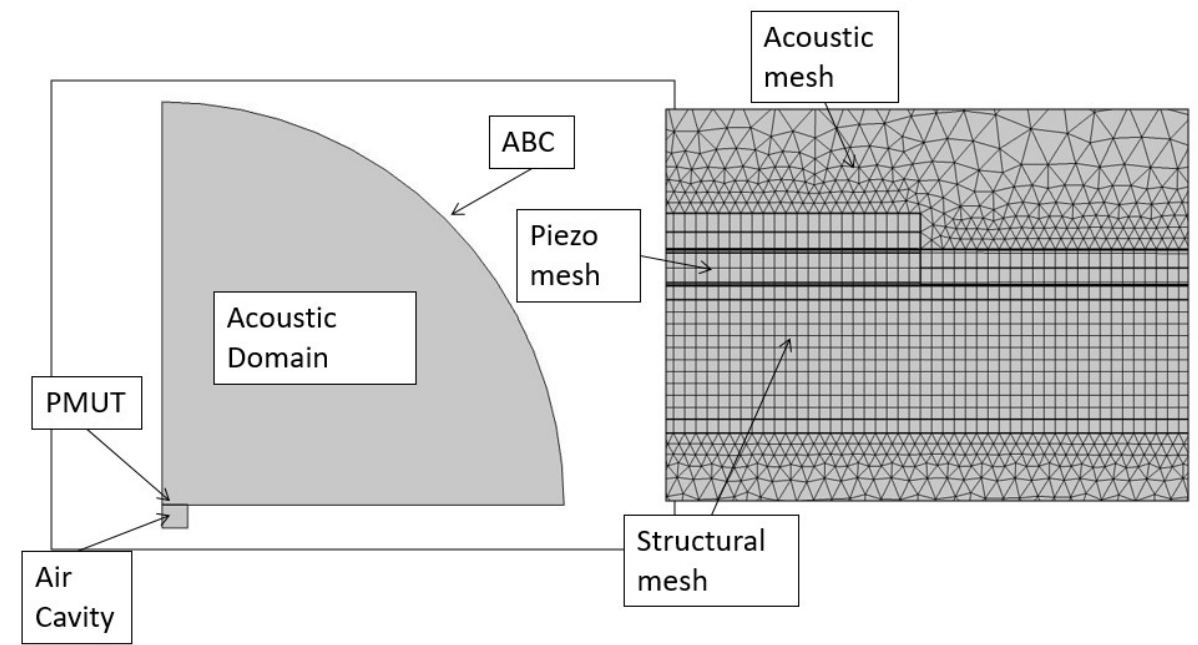

Figure 2: 2D axial-symmetric single PMUT model (left); PMUT and acoustic mesh detail: 3-node triangular elements, 4-node quadrilateral elements (right).

correspondence of the performing fundamental electro-mechanical eigenmode. Furthermore, the radiation into an infinite medium is simulated by means of the COMSOL absorbing boundary conditions (ABC), adopted on the acoustic circular exterior boundary, while a rigid hard wall condition is imposed on the surfaces of the air cavity and on the remaining acoustic boundary.

The initial deflected configuration of the system, due to the residual stress state, is correctly captured, including the geometric non-linear effects by means of the adopted large displacements formulation. Additionally, the transducer non-linear dynamic EMA response is computed under a sinusoidal voltage input excitation at the previously estimated linearized fundamental electro-mechanical frequency $f_{0}[21,22,23,24$. At this stage, the linear acoustic behavior is considered for air, therefore, the non-linearities only refer to the employed large displacements formulation. To solve the problem, the generalized alpha implicit time integration scheme is adopted with a time step equal to $T_{0} / 64$, where $T_{0}=1 / f_{0}$. Finally, the vertical spatial mean acceleration time history are extracted at the acoustic-structure interaction surface.

In the second model, the behaviour of the complete set of PMUTs, arranged 
in the $4 \times 4$ array configuration, is simulated. This system is characterized by two orthogonal symmetry planes, passing through the device center (see a detail in Fig. 33.

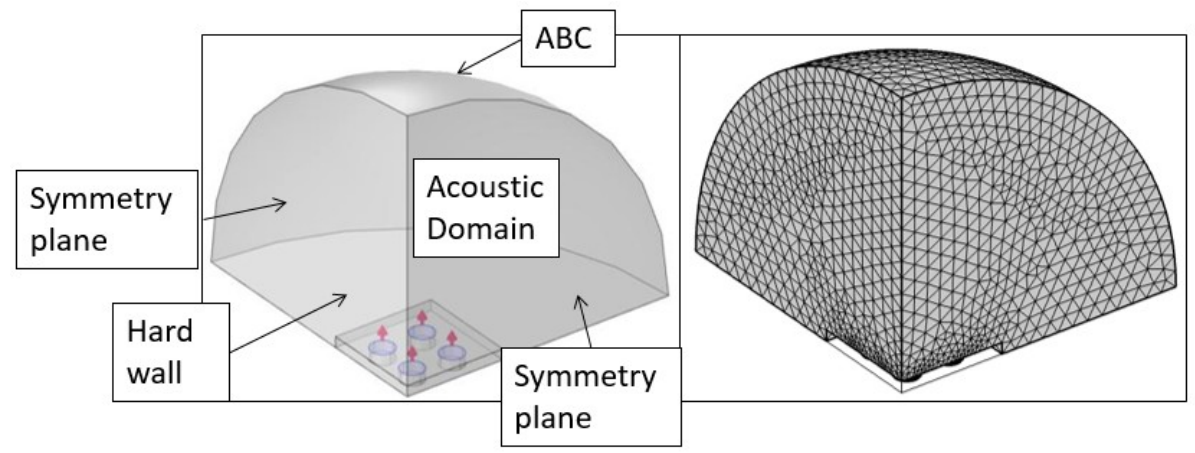

Figure 3: Acoustic 3D model, a quarter of a 4x4 array of PMUTs with imposed vertical acceleration on the equivalent pistons (red arrows); acoustic mesh detail: 10node tetrahedral elements (right).

The linear acoustic 3D problem is solved in a quarter of a hemispherical nondissipative air domain [18, 25], with a radius of $3 \lambda$, considering the presence of a quarter of silicon die with full size equal to $7.2 \times 7.2 \times 0.4 \mathrm{~mm}^{3}$.

Accordingly, PMUTs belonging to a $2 \times 2$ set are modelled as equivalent vibrating baffled rigid pistons, on the die surface. They are characterized by the same diaphragm area of the real transducers. The displacement of the pistons is enforced by assigning the history of the spatial mean of the vertical acceleration, previously computed by means of the 2D EMA model for the complete PMUT stack. Also in this case, the absorbing boundary conditions (ABC) are considered on the acoustic spherical boundary in order to simulate the radiation into an infinite medium. Additionally, the symmetry boundary condition is enforced on the two vertical planes while a hard wall condition, which corresponds to zero normal acoustic accelerations, is imposed on the bottom plane. The computational domain consists of quadratic tetrahedra with a maximum elements size equal to $\lambda / 6=572.33 \mu \mathrm{m}$. Additionally, a proper refinement is adopted near the acoustic sources so that the circular piston area is discretized using 10 
a total number of degrees of freedom equal to 300139.

The array response is evaluated in the acoustic domain by means of the generalized alpha implicit time integration scheme with a time step equal to $T_{0} / 32$, where $T_{0}=1 / f_{0}$. Hence, the results are computed in terms of pressure 145 maps and time histories along the vertical acoustic axis passing from the center of the device.

\section{Results and experimental validation}

The EMA single PMUT FEM model correctly estimates the initial deflection and the linearized electro-mechanical fundamental eigenfrequency of the system. The measured initial vertical displacement of the center of the plate is equal to $5.2 \mu \mathrm{m}$ (Fig. 4). The numerical prediction, on the other hand, is equal to 4.9 $\mu \mathrm{m}$. Due to the residual stresses, a substantial shift of the fundamental mode eigenfrequency is registered: it passes from $111.5 \mathrm{kHz}$, without the influence of the initial stress state, to $100.0 \mathrm{kHz}$ (see Fig. 10. The uncertainty in the values of the residual stresses of the PMUT layers, determines the slight mismatch in the initial configuration.

In order to investigate the influence of the static voltage bias on the PMUT deflection, an experimental campaign, by means of Polytec MSA-500 (Fig. 5), has been carried out on 16 PMUTs. The initial deformed profiles along a diameter, measured through the white-light interferometry, are shown in Fig. 6 where the PZT hat layer appears about $2 \mu \mathrm{m}$ thick, instead of $1.06 \mu \mathrm{m}$, due to the silicon refractive index compared to the top electrode one. It is worth noting that, because of the noise of the measure, a filter has been applied to the case of $V_{\text {bias }}=0 \mathrm{~V}$, to get a smoother profile, as a consequence no jump in the PZT thickness appears.

The topography of each membrane and the displacement profile, along the diameter of the transducer, has been extracted. The test shows how the application of a constant bias voltage affects the initial static configuration (Fig. 6). 


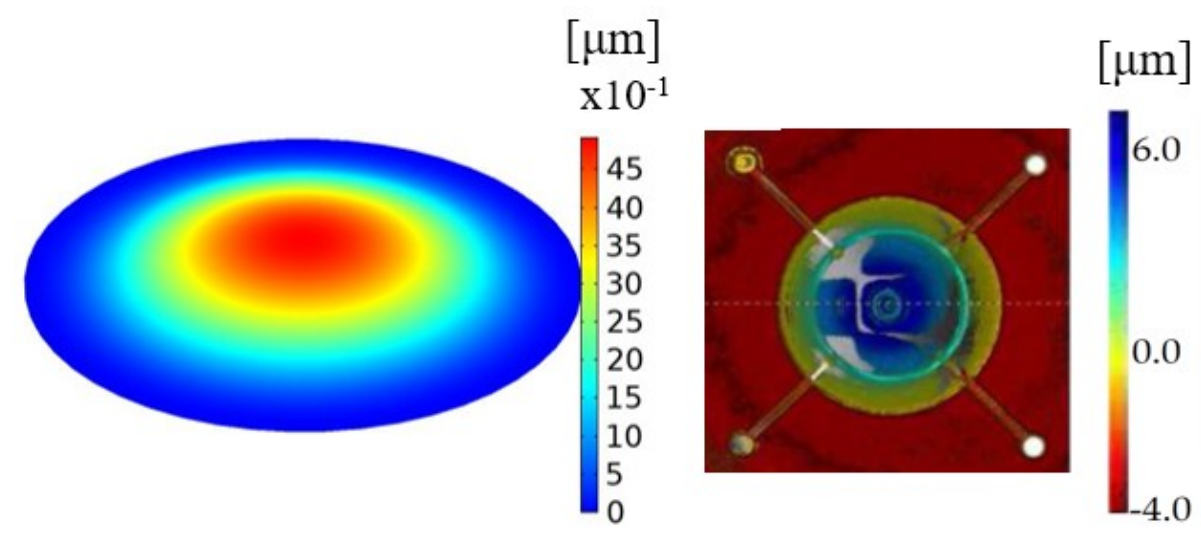

Figure 4: Initial static transversal displacement in $\mu \mathrm{m}$ : numerically computed by means of COMSOL Multiphysics 5.4 (left); measurement by means of Polytec MSA500, top view (right);

The vertical static displacement at the center of the PMUTs has been measured and plotted at different constant bias voltages. Fig. 7 (on the left) shows the initial vertical displacement of each transducer, in correspondence of $V_{\text {Bias }}=0 \mathrm{~V}$, is in the upward direction and falls within a range of $3.5 \mu \mathrm{m}$ to $5.5 \mu \mathrm{m}$. The differences between the various curves are related to the spatial distribution of the residual stress state on the wafer.

Increasing the value of the constant bias voltage, starting from $0 \mathrm{~V}$ to 20 $\mathrm{V}$, the value of the vertical displacement at the center of the transducer decreases. The displacement becomes negative and the initial deflected configuration changes concavity (as shown in Fig. 6).

Going further, the fundamental frequency of the system has been measured by means of the Polytec MSA-500 laser-doppler vibrometer applying a pseudorandom excitation. Fig. 7 reports that the resonance frequency decreases reaching the minimum value between $14-16 \mathrm{~V}$. The mechanical explanation of this phenomenon is related to the fact that the application of the constant voltage bias determines an internal bending moment that compensates the bending moment due to the initial stress, bringing the system back to the undeformed 


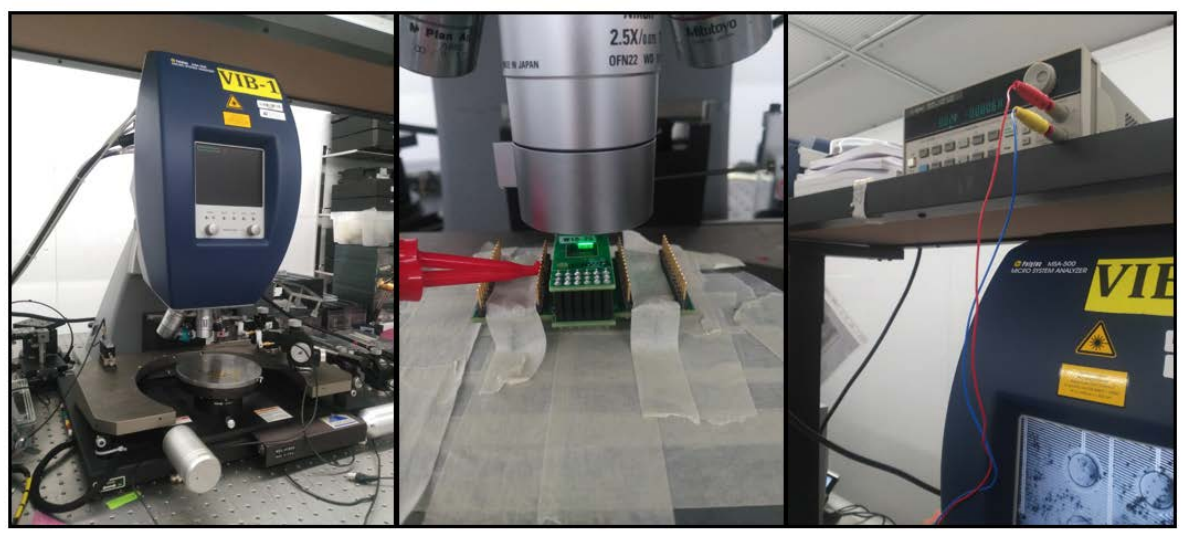

Figure 5: Polytec MSA-500 (left); setup for the time histories measurements (center); external generator for the actuation of the membranes (right).
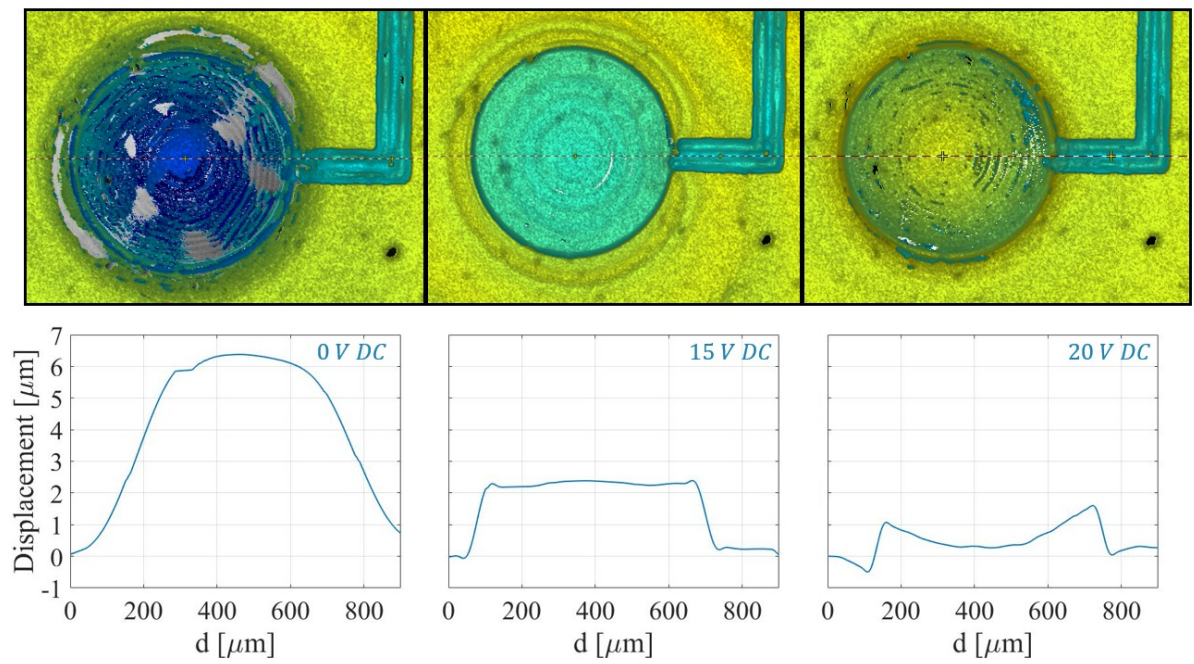

Figure 6: Topography of one membrane of the array for different constant bias voltages: $0 \mathrm{~V}$ DC (top left), $15 \mathrm{~V} \mathrm{DC}$ (top center), $20 \mathrm{~V} \mathrm{DC} \mathrm{(top} \mathrm{right);} \mathrm{initial} \mathrm{static} \mathrm{transversal}$ displacement in $\mu \mathrm{m}$ : experimental measurement by means of Polytec MSA-500 for each case (bottom). 
reference flat configuration. Through this process, the stiffness of the system decreases, due to the fact that the stress resultant due to the voltage bias load is negative and it reaches its minimum values when the diaphragm is flat. By increasing the voltage beyond this point, the system goes downward, the reverse sign deformation appears in the plate cross-section, subsequently the stress resultant due to the voltage bias load increases and the stiffness starts increasing as well as the PMUT fundamental frequency.
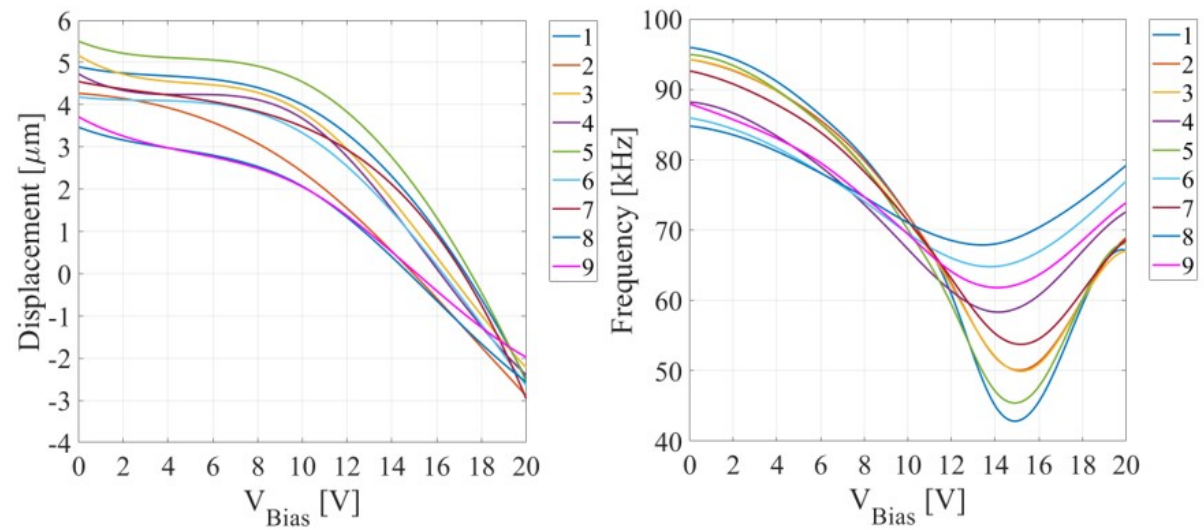

Figure 7: Experimental measurements: constant bias voltage influence on the vertical displacement (left); constant bias voltage influence on the resonance frequency (right).

Hence, the application of a constant voltage difference to the PZT electrodes yields an imposed stress distribution that determines the deformed configuration. Considering a set of 9 measured diaphragms, the trend in Fig. 7 shows that the reversed static configuration of the plate occurs by increasing the voltage beyond a specific threshold for each PMUT, in the range of $15 \mathrm{~V}$ to $17.5 \mathrm{~V}$. The fundamental resonance frequency of the system is affected by the membrane stresses [26, 23, 27] by means of the geometric stiffness. The initial stress state, in correspondence of $V_{\text {Bias }}=0 \mathrm{~V}$ at the center of the membrane, is characterized by a negative membrane stress resultant that becomes larger and larger, increasing the voltage while the system tends to the flat configuration. Hence, the fundamental frequency decreases with the voltage until the diaphragm approaches the flat configuration. By increasing the voltage beyond the flat threshold, the 
diaphragm shows the reversed configuration and consequently the opposite deformation state. Going further, the stress resultant at the PMUT center due to the applied voltage bias increases as well as the fundamental frequency, while the displacement becomes larger and oriented downward. A set of analyses in merical results in terms of pressure time histories with $2 \mathrm{~V}$ DC constant bias voltage. The time histories of pressure have been measured through the Brüel \& Kjær 1/8" microphone type 4138 with sensitivity equal to $89.12 \mathrm{mV} / \mathrm{Pa}$ at $100 \mathrm{kHz}$. The numerical and the experimental results are in good agreement. 225 In particular the non-linearity threshold voltage amplitude is $300 \mathrm{mV}$.

Once the non-linearity shows up, the amplitude modulation appears. This phenomenon is due to the fact that the excitation frequency is kept fixed at the linearized resonance frequency while the real resonance frequency of the system depends on the oscillation amplitude, in the non-linear regime, and it slightly $300 \mathrm{mV}$. It is worth noting that, with the application of the static voltage bias of $2 \mathrm{~V}$ the non-linear phenomenon occurs in correspondence of lower $V_{A C}$ than the values previously reported in [16]. As a matter of fact, the imposed constant voltage reduces the stiffness of the system. As a consequence, the 235 stiffness, increases. 

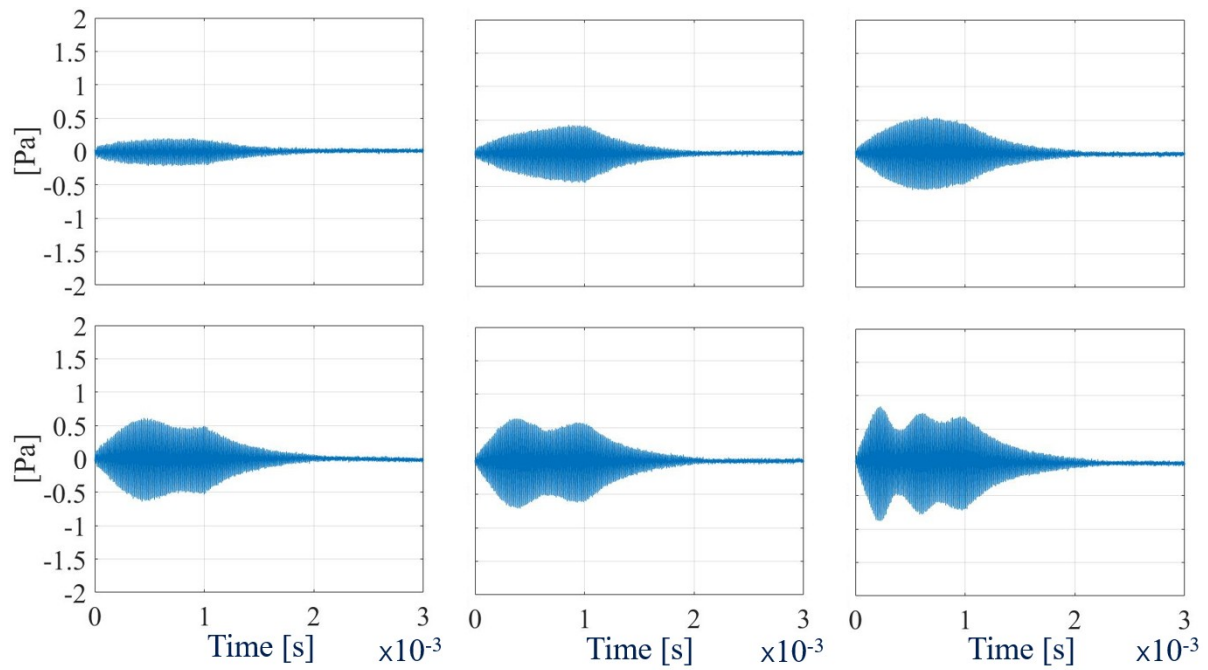

Figure 8: Experimental pressure time histories at $3 \mathrm{~cm}$ on the vertical acoustic axis of the transducer. $V_{A C}=100 \mathrm{mV}$ (top left), $200 \mathrm{mV}$ (top center), $300 \mathrm{mV}$ (top right), $400 \mathrm{mV}$ (bottom left), $500 \mathrm{mV}$ (bottom center), $1 \mathrm{~V}$ (bottom right).
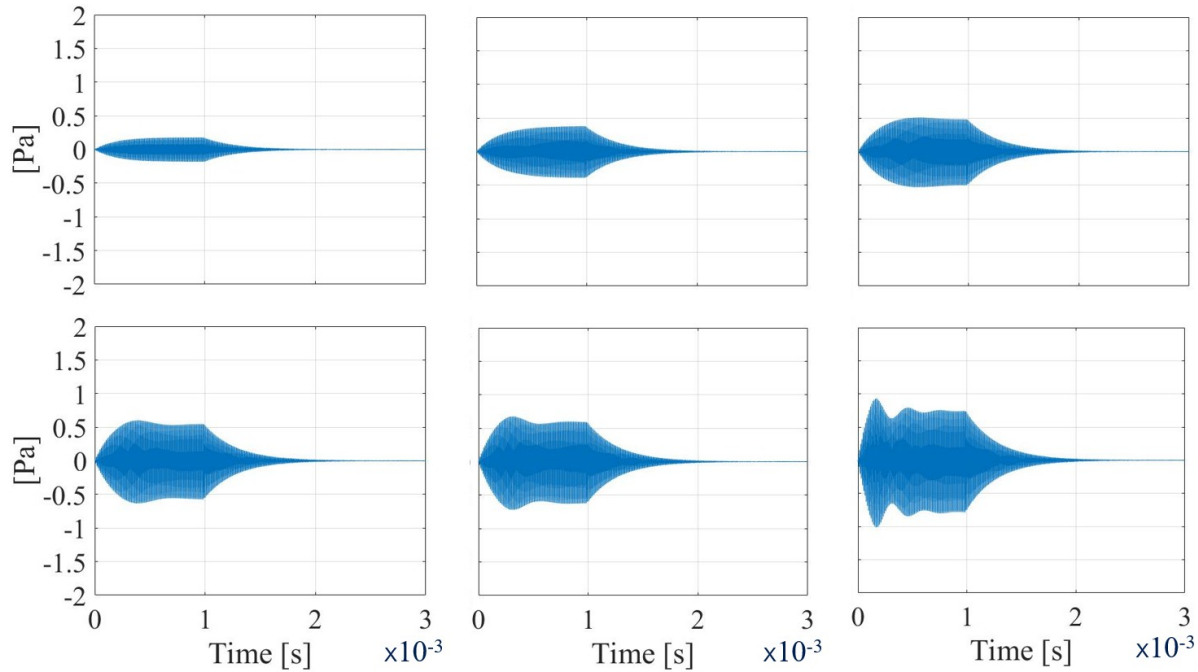

Figure 9: Numerical pressure time histories at $3 \mathrm{~cm}$ on the vertical acoustic axis of the transducer. $V_{A C}=100 \mathrm{mV}$ (top left), $200 \mathrm{mV}$ (top center), $300 \mathrm{mV}$ (top right), $400 \mathrm{mV}$ (bottom left), $500 \mathrm{mV}$ (bottom center), $1 \mathrm{~V}$ (bottom right). 
The damping property of the system has been measured through a free vibration decay test. In particular the total quality factor, associated with the first mode, is equal to $Q_{t o t}=74$. Generally, several sources of energy losses sum

of time domain vibrometer measures through the Polytec MSA-500. The experimental procedure consists in collecting the steady-state vertical displacement 


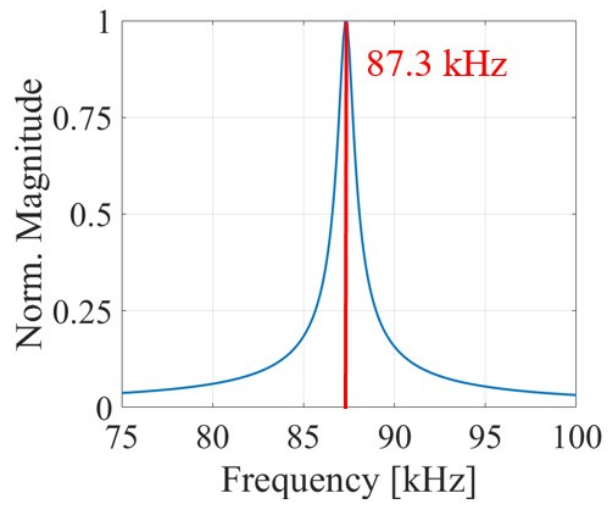

Figure 10: Transversal displacement normalized amplitude frequency spectrum, at the PMUT center. Numerically computed by means of COMSOL Multiphysics 5.4

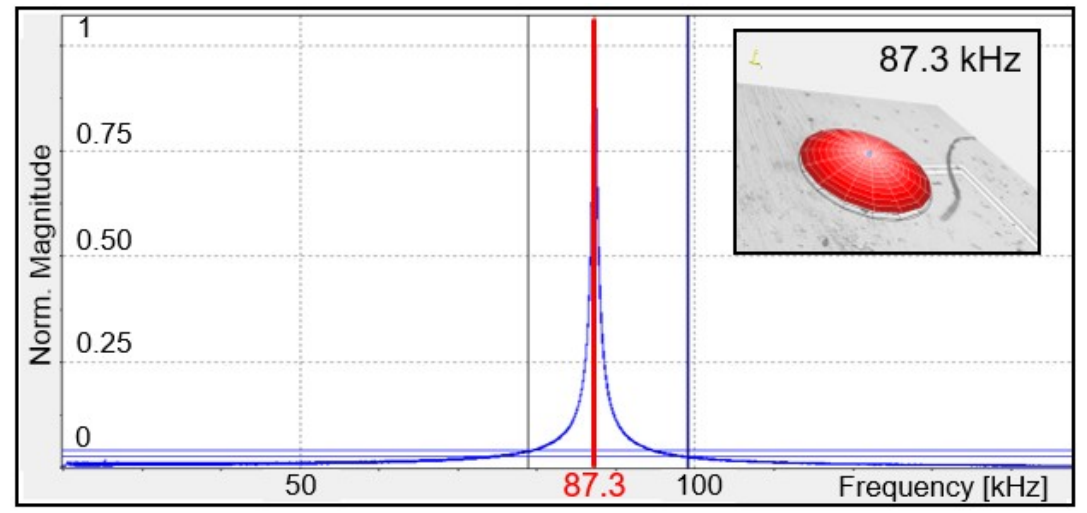

Figure 11: Transversal displacement normalized amplitude frequency spectrum, at the PMUT center. Experimentally measured by means of Polytec MSA-500; first mode shape of the transducers recorded by means of Polytec MSA-500 (inset). 
oscillation amplitude, sweeping up the excitation input frequency at different voltage amplitude $V_{A C}$. Hence, the frequency response functions (FRFs) in terms of displacement amplitudes at different input frequencies have been reconstructed and reported in Fig. 12. The linearized fundamental resonance frequencies have been previously measured by means of pseudo-random vibration tests (see Figs. 7 on the right and 11).
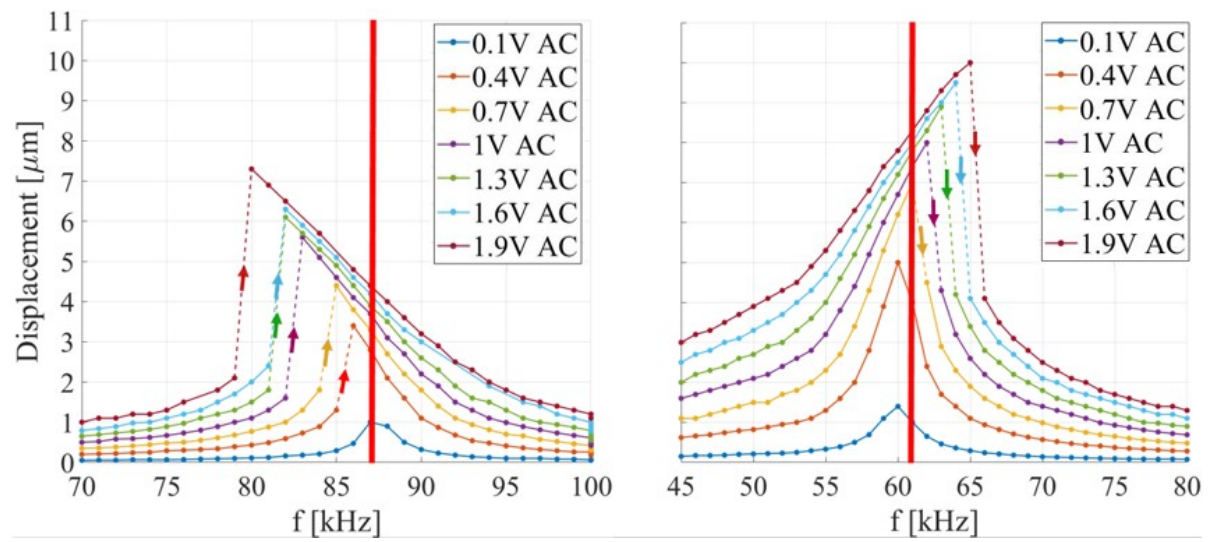

Figure 12: Experimental soft-spring curve: $V_{D C}=2 \mathrm{~V}, V_{A C}$ sweep. Red vertical line is associated with the linearized resonance frequency $f_{0}=87.3 \mathrm{kHz}$ (left); experimental hard-spring curve: $V_{D C}=12 \mathrm{~V}, V_{A C}$ sweep. Red vertical line is associated with the resonance frequency $f_{0}=61.3 \mathrm{kHz}$ (right). Arrows refer to the jump direction.

Fig. 12 shows the effect of increasing the $V_{A C}$ excitation voltage amplitude at two different constant bias voltages. Focusing the attention on the graph for $V_{D C}=2 \mathrm{~V}$ (left), the system, for low values of $V_{A C}$ shows a linear behavior and the maximum value occurs very close to the linearized measured resonance frequency. Increasing the excitation amplitude above $V_{A C}=400 \mathrm{mV}$, the nonlinear soft spring behavior arises and a jump in the amplitude appears. For the case of $V_{D C}=12 \mathrm{~V}$, the relative mismatch between the reported resonance frequency of $61.3 \mathrm{kHz}$ and the peak frequency of $60.1 \mathrm{kHz}$ is less than $2 \%$ : such a discrepancy might be attributed to a too fast measurement technique that is not allowing for the vibration amplitudes to settle to the steady state. It is worth noting that, in the case of $V_{D C}=12, \mathrm{~V}$ the non-linear hardening behaviour 
occurs above the voltage threshold of $V_{A C}=700 \mathrm{mV}$.

This behavior is related to the influence of the vertical oscillation amplitude, on the stiffness of the pre-stressed and pre-deflected system, as it happens in the large bending vibrations of the initially stressed shallow shells and arches [14, 33, 34, 35, 36]. As the vertical oscillation becomes relevant, it affects the stiffness and consequently the resonance frequency of the system subsequently the initial amplitude modulated transient vibrations occur, as reported in the measured pressure histories in Fig. 8, resulting in transverse displacement FRFs which depend on the displacement amplitude.

Considering the transducer as a circular layered plate [37] with radius $R$, we report the weak formulation of the electromechanical problem, in the transmission phase. To this scope, the Kirchhoff kinematics and the axisymmetric hypothesis are exploited, considering each layer as a linear elastic isotropic material but for the PZT layer, in which an isotropic linear stress-charge constitutive law is employed. Hence, the influence on the transverse oscillation $w(r, t)$ dial residual stress $\sigma_{r 0}$ are taken into account, by means of the adopted large displacement formulation [24]. Furthermore, the effect of a uniform alternate electric field $E_{A C}$ and the acoustic pressure load $p_{a c}$ are considered.

Assuming $w_{0}$ as the equilibrium configuration, under the pre-stress state and 
find $w(r, t) \in \mathcal{C}(0)$, such that

$$
\begin{aligned}
& \int_{0}^{R} \bar{\rho} \frac{\partial^{2} w}{\partial t^{2}} \delta w r d r \\
+ & \int_{0}^{R} N_{r}^{\sigma_{0}}\left(\frac{\partial w}{\partial r}-\frac{\partial w_{0}}{\partial r}\right) \frac{\partial \delta w}{\partial r} r d r+\int_{0}^{R} \bar{e} E_{D C}\left(\frac{\partial w}{\partial r}-\frac{\partial w_{0}}{\partial r}\right) \frac{\partial \delta w}{\partial r} r d r \\
+ & \int_{0}^{R} D\left(\frac{\partial^{2} w}{\partial r^{2}}-\frac{\partial^{2} w_{0}}{\partial r^{2}}\right) \frac{\partial^{2} \delta w}{\partial r^{2}} r d r+\int_{0}^{R} D \frac{1}{r}\left(\frac{\partial w}{\partial r}-\frac{\partial w_{0}}{\partial r}\right) \frac{\partial \delta w}{\partial r} d r \\
+ & \int_{0}^{R} F\left(\frac{\partial w}{\partial r}-\frac{\partial w_{0}}{\partial r}\right) \frac{\partial^{2} \delta w}{\partial r^{2}} d r+\int_{0}^{R} F\left(\frac{\partial^{2} w}{\partial r^{2}}-\frac{\partial^{2} w_{0}}{\partial r^{2}}\right) \frac{\partial \delta w}{\partial r} d r \\
+ & \int_{0}^{R} \frac{3}{2} \bar{E}\left(\frac{\partial w_{0}}{\partial r}\right)^{2}\left(\frac{\partial w}{\partial r}-\frac{\partial w_{0}}{\partial r}\right) \frac{\partial \delta w}{\partial r} r d r \\
+ & \int_{0}^{R} \frac{3}{2} \bar{E}\left(\frac{\partial w_{0}}{\partial r}\right)\left(\frac{\partial^{2} w}{\partial r^{2}}-\frac{\partial^{2} w_{0}}{\partial r^{2}}\right)^{2} \frac{\partial \delta w}{\partial r} r d r \\
+ & \int_{0}^{R} \frac{1}{2} \bar{E}\left(\frac{\partial w}{\partial r}-\frac{\partial w_{0}}{\partial r}\right)^{3} \frac{\partial \delta w}{\partial r} r d r= \\
= & \int_{0}^{R} \psi E_{A C}\left(\frac{\partial \delta w}{\partial r}\right) r d r+\int_{0}^{R} \zeta E_{A C}\left(\frac{\partial \delta w}{\partial r}\right) d r-\int_{0}^{R} p_{a c} \delta w r d r
\end{aligned}
$$$$
\forall \delta w \in \mathcal{C}(0)
$$

with

$$
\begin{gathered}
\mathcal{C}(0)=\{v \mid v \text { sufficiently regular in }(0, R) \text { and } v(0)=0, v(R)=0, \\
\left.\frac{\partial v}{\partial r}(0)=0, \frac{\partial v}{\partial r}(R)=0\right\}
\end{gathered}
$$


Moreover, the following equalities hold:

$$
\begin{aligned}
& \bar{\rho}=\sum_{k=1}^{n} \int_{t_{k-1}}^{t_{k}} \rho d z \\
& N_{r}^{\sigma_{0}}=\sum_{k=1}^{n} \int_{t_{k-1}}^{t_{k}} \sigma_{r 0} d z \\
& \bar{e}=-\sum_{k=1}^{n} \int_{t_{k-1}}^{t_{k}} e_{31} d z \\
& D=\sum_{k=1}^{n} \int_{t_{k-1}}^{t_{k}} \frac{z^{2} E}{1-\nu^{2}} d z \\
& F=\sum_{k=1}^{n} \int_{t_{k-1}}^{t_{k}} \frac{z^{2} \nu E}{1-\nu^{2}} d z \\
& \bar{E}=\sum_{k=1}^{n} \int_{t_{k-1}}^{t_{k}} \frac{E}{1-\nu^{2}} d z \\
& \psi=-\sum_{k=1}^{n} \int_{t_{k-1}}^{t_{k}} z e_{31} d z \\
& \zeta=-\sum_{k=1}^{n} \int_{t_{k-1}}^{t_{k}} z e_{32} d z \\
& E_{D C}=-\frac{V_{D C}}{t_{p z t}} \\
& E_{A C}=-\frac{V_{A C}(t)}{t_{p z t}} \\
& F_{2}
\end{aligned}
$$

in which $\rho$ is the mass density of the system, $e_{31}$ is the piezoelectric coupling coefficient, $\mathrm{E}$ and $\nu$ are the Young's modulus and the Poisson ratio, $n$ is total number of layer and $t_{k}$ is the thickness of the k-th layer. Furthermore, the following inequality holds:

$$
\frac{t_{p z t}}{R}<<1
$$

Hence, it is reasonable to approximate the transverse component of the electric field, in the piezoelectric layer, with the one of a capacitor with thickness $t_{p z t}$, with a good accuracy but for the cylindrical lateral boundary [38. Therefore, assuming $V_{D C}$ and $V_{A C}(t)$ respectively the static and the alternate voltage, 
applied to the top electrode of the piezoelectric layer, the static and alternate electric fields become:

$$
E_{D C}=-\frac{V_{D C}}{t_{p z t}} \quad E_{A C}=-\frac{V_{A C}(t)}{t_{p z t}}
$$

Considering (1), the non-linear trend is determined by the contribution to the linear stiffness of the internal membrane axial force, associated with the linearized resonance frequency. The axial force is induced by the combination of the residual stresses and the static voltage $V_{D C}$ load. Furthermore, assuming the large displacement formulations in the elastodynamics equations, the initial deformed reference equilibrium configuration influences the non-linear second order stiffness terms, around which the system oscillates and it is induced by the combination of the pre-stress state and the static voltage, as well. Moreover, the amplitude of transverse motion affects the third order non-linear stiffness term. The combination of the two non-linear stiffness terms determines the type of non-linear trend in the FRF. Furthermore, the third order stiffness term can be considered as a source of hardening in the FRF while the second order one as a source of softening. More precisely, the latter contribution becomes dominant beyond a certain threshold of the ratio between the initial transverse deflection and the oscillation steady state amplitude [39, 40]. Finally, the acoustic pressure $p_{a c}$ play a role on the stiffness as well. As a matter of fact, it increases the mass term and, consequently, it affects the stiffness. The phenomenon is due to its proportionality to the accelerations, by means of the acoustic-structural coupling condition 18

$$
\frac{\partial p_{a c}}{\partial z}=-\rho_{f} \ddot{w}
$$

where $\rho_{f}$ is the fluid mass density.

Considering the case of $V_{D C}=2 \mathrm{~V}$, characterized by an upward initially deflected system, the combination of the non-linear stiffness terms implies the softening behaviour (refer to Fig. 12 on the left). Concerning the case in Fig. ${ }_{310} 12$ on the right, related to constant bias voltage $V_{D C}=12 \mathrm{~V}$, the associated initial deflection is close to the flat configuration, as it is shown in Fig. 7. As 
a matter of fact, at the center of the PMUT the membrane stress resultant due to the static voltage is compressive and, for an almost flat configuration, that entails the reduction of the stiffness and of the linearized resonance frequency. longing to the die. This reduces each vibrating transducer to an oscillating rigid baffled piston.

For the case of $V_{A C}=100 \mathrm{mV}$ TX actuation, the numerical pressure maps at $t=1.02 \mathrm{~ms}$, i.e. just before the excitation stops, are shown on the top part of 340 Fig. 14. Additionally the numerical pressure time histories are reported, on the bottom part of Fig. 14, at the point P, placed on the vertical acoustic axis which crosses the center of the system at the distance of $2 \lambda$. As it can be noticed, the 

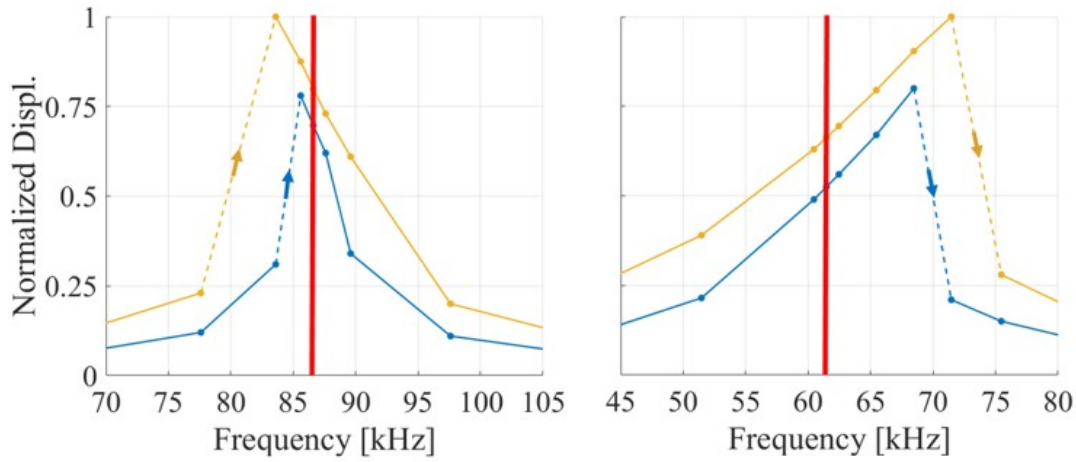

Figure 13: Numerical soft-spring curve: $V_{D C}=2 \mathrm{~V}, V_{A C}=700 \mathrm{mV}$ (blue line), $V_{A C}=1.4 \mathrm{~V}$ (yellow line). Red vertical line is associated with the linearized resonance frequency $f_{0}=87.3 \mathrm{kHz}$ (left); numerical soft-spring curve: $V_{D C}=12 \mathrm{~V}, V_{A C}=700$ $\mathrm{mV}$ (blue line), $V_{A C}=1.4 \mathrm{~V}$ (yellow line). Red vertical line is associated with the resonance frequency $f_{0}=61.3 \mathrm{kHz}$ (left). Arrows refer to the jump direction.

wave front is flat close to the die and become spherical at large distance.

It is interesting to compare this results in order to evaluate the performance of the in parallel actuation of the complete set of $4 \times 4$ PMUTs with respect to the single transducer one.

It is worth noting that, in the array case, the pressure amplitude is 10 times higher than the case of the single diaphragm. This can be attributed to the fact that 16 transducers are active and the wave front tends to the spherical configuration for which the $1 / r$ decreasing pressure amplitude relation holds, at a larger distance with respect to that one related to the wave front created by the single transducer actuation. This is confirmed by the corresponding pressure map at the time instant $t=1.02 \mathrm{~ms}$ represented in Fig. 14 on top right, where the pressure propagation becomes rapidly spherical.

To investigate the simulation capability of the $4 \times 4$ array model, different kinds of actuation have been considered, as illustrated in Fig. 15. The actuation type affects the pressure maps in the acoustic domain and each case is associated with a proper propagation pattern reported in Fig. 16 .

The considered acoustic domain has radius equal to $9 \lambda$ and the numeri- 

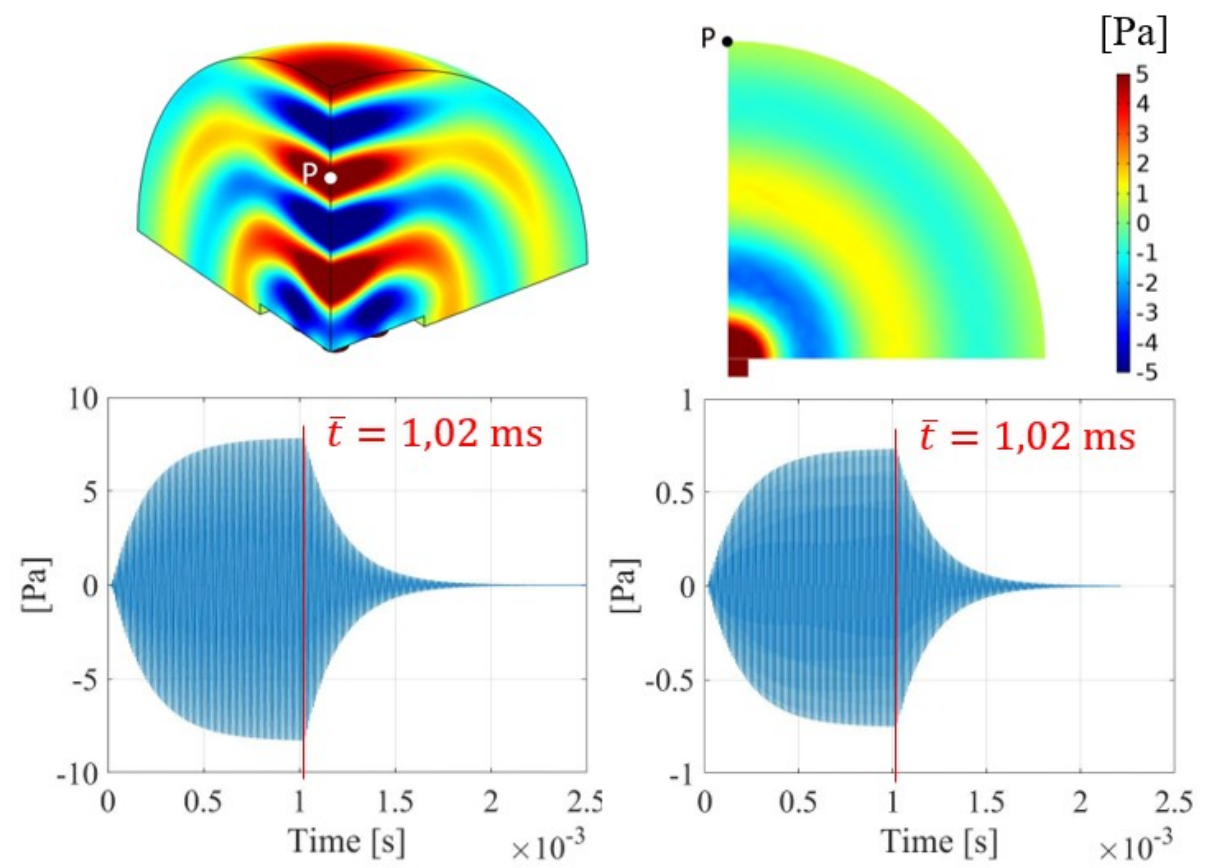

Figure 14: Numerical pressure map [Pa] at $t=1.02 \mathrm{~ms}$ : 3D acoustic $4 \mathrm{x} 4$ array model (top left), 2D axisymmetric PMUT EMA model (top right). Numerical pressure time histories at point $\mathrm{P}=(0,0,2 \lambda)$ : $3 \mathrm{D}$ acoustic $4 \mathrm{x} 4$ array model (bottom left), $2 \mathrm{D}$ axisymmetric PMUT EMA model (bottom right). 

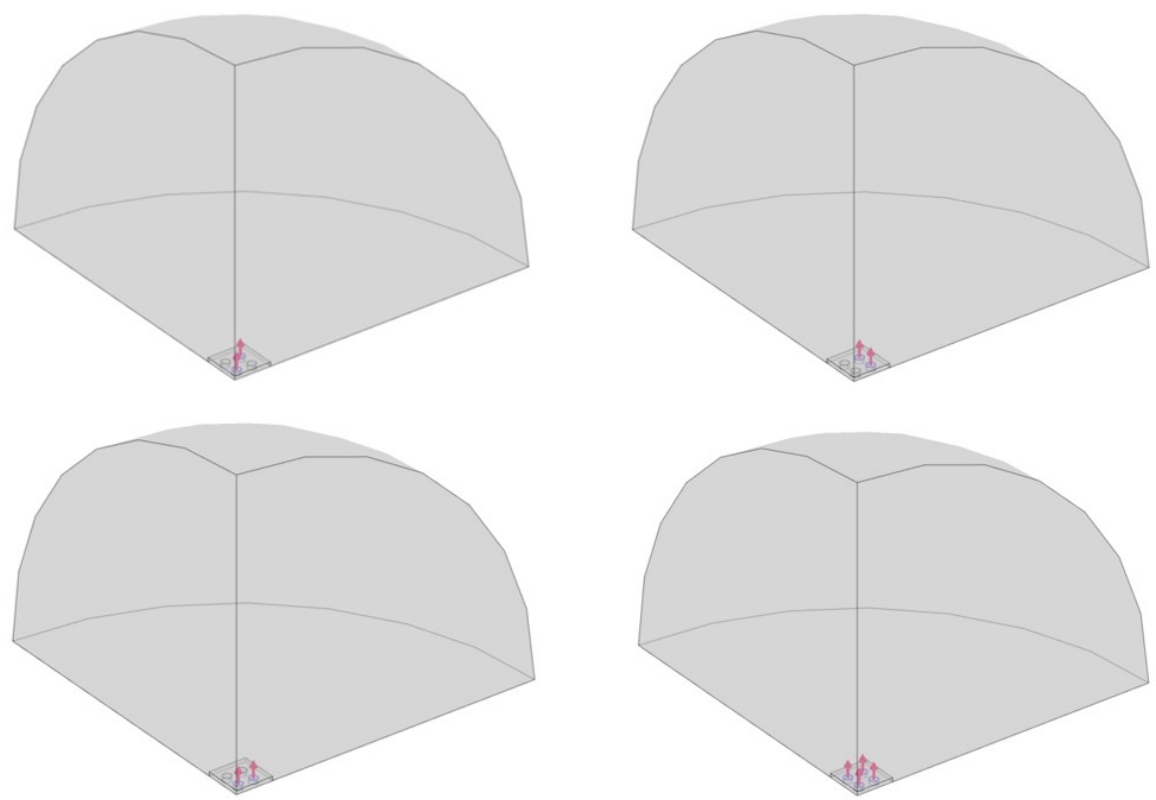

Figure 15: Different cases studies: diagonal actuation (top left), external actuation (top right), internal actuation (bottom left), delayed actuation (bottom right) 
cal pressure time histories evaluated at the point $\mathrm{P}=(0,0,9 \lambda)$ belongs to the acoustic boundary, are extracted. Hence, the pressure comparison is reported in Fig. 17 .
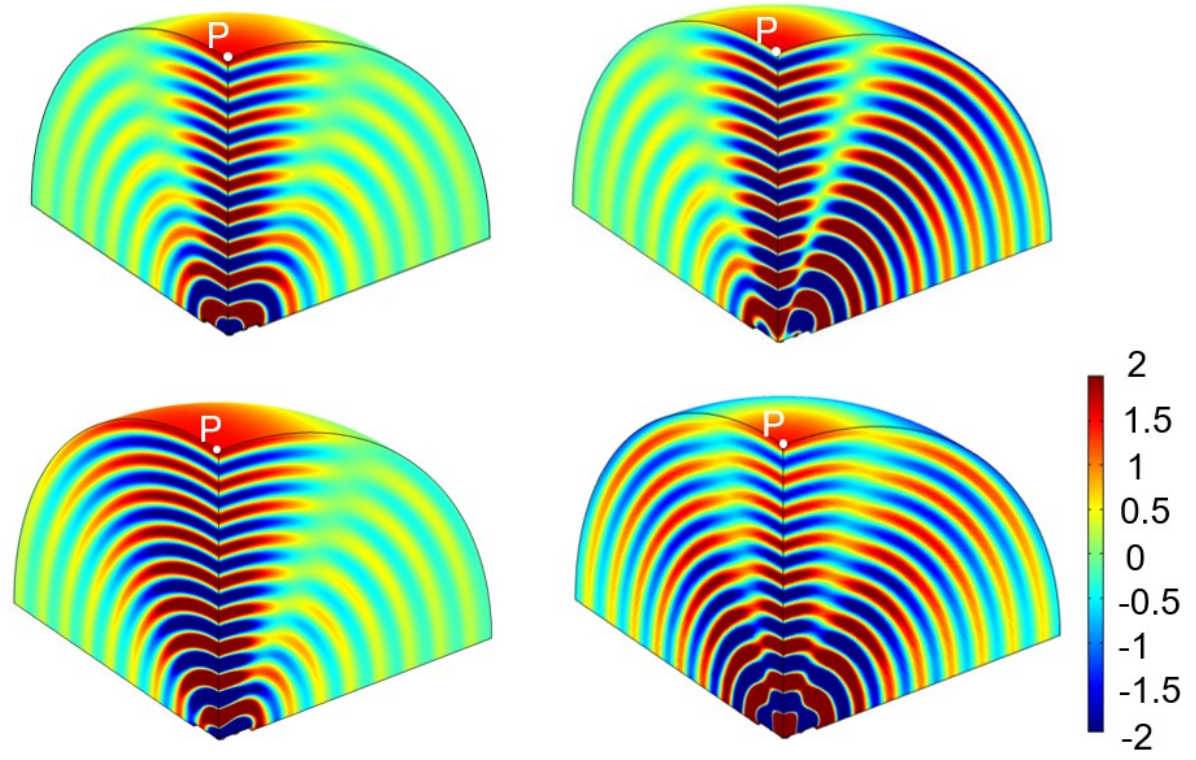

Figure 16: Pressure map for the different cases study: a) diagonal actuation (top left), b) external actuation (top right), c) internal actuation (bottom left), d) delayed actuation (bottom right)

The computed pressure time histories at the point $\mathrm{P}$ are similar. The vertical acoustic axis of the transducer, passing through the die centre, always represents one of the preferential propagation direction, due to the transverse diaphragms oscillation. On the other hand, the actuation procedure strongly affects the 3D propagation. Consequently, the pressure maps are deeply different on the other directions as shown in Fig. 16. The model correctly captures the initial rest time, corresponding to the so-called time of flight (TOF), that represents the time a wave takes to cover the distance between the acoustic source and the pressure evaluation point. The case a) results in a slightly higher steady state pressure than the other ones. This is due to the fact that, accordingly the analyzed actuation, the acoustic waves mainly propagate along the vertical axis 

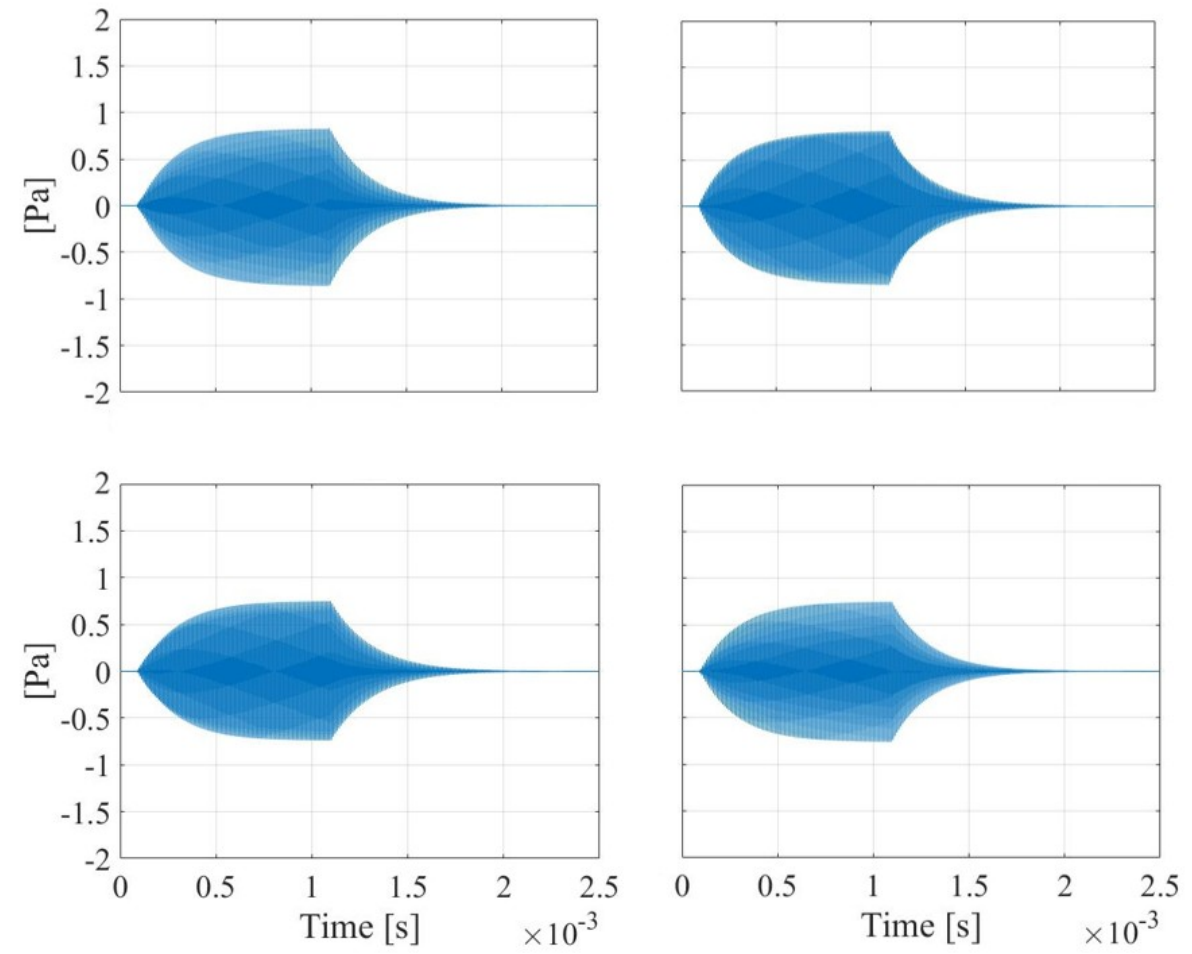

Figure 17: Pressure time history at point $\mathrm{P}(0,0,9 \lambda)$ for the different cases study: diagonal actuation (top left), external actuation (top right), internal actuation (bottom left), delayed actuation (bottom right) 
of the device with the presence of side lobes of propagation.

This procedure can be used to preliminarily assess the wave propagation coming from an array of oscillating transducers. Therefore, the presented approach represents a suitable technique to solve beamforming and steering acoustic problems.

\section{Closing remarks}

This work presents the results obtained by means of a two stages simulation procedure involving two proper FEM models of Piezoelectric Micromachined Ultrasonic Transducers (PMUTs), at two different modelling scales.

In the first one the fully coupled ElectroMechanical-Acoustics problem (EMA) is solved considering the single diaphragm behaviour. At this stage the response is obtained in the time domain, account taken the non-linear involved dynamics, by means of the adopted large displacement formulation. Furthermore, the results of an experimental campaign in the mechanical and acoustic domains, for the air-coupled PMUT, are reported. In particular, static interferometric and dynamic vibrometric measurements are presented. Therefore, the effect of the residual stresses and the DC voltage bias influence, on the PMUT deflected configuration and the resonance frequency, has been thoroughly investigated. The experiments show the fundamental frequency variation and the concavity change of the reference configuration, due to the static voltage load. Moreover, the non-linear displacement frequency response functions (FRFs) have been evaluated, considering a set of applied AC voltage input amplitudes and DC bias voltages. The observed phenomena are exhaustively described and proper mechanical explanations are provided. Thus, the FEM PMUT fully coupled non-linear model successfully captures the analysed experimental trends.

The influence of non-linearity on the diaphragm vibration is studied in the first stage, providing the linearity limit in the transducer dynamic behaviour. As a matter of fact, loading the system with an increasing voltage at the fixed linearized fundamental electromechanical resonance frequency, the recorded ex- 
perimental and the numerical pressure histories show a initial non-linear amplitude modulated dynamic behavior. Hence, the proposed single transducer model represents a suitable tool to estimate the non-linear coupled response and subsequently the non-linear amplitude voltage threshold, over which any linear equivalent transducers model fails.

As the main result of this simulations, the coupled acceleration time history is extracted and subsequently the spatial mean history over the acousticstructure interaction surface is computed. Furthermore, the $4 \times 4$ PMUTs array performance is simulated through the second FEM 3D model. As a matter of fact, the pressure propagation and beamforming problem is solved by means of a 3D acoustic model of the complete set of transducers. At this final stage, the equivalent piston-like modelling of the transducers is considered. As the result of the 3D acoustic array simulation, the device performance is studied considering different kinds of actuation and the single PMUT behaviour is compared to the $4 \times 4$ PMUTs array response.

Work in progress includes the numerical simulations of different beamforming and the RX phases, including the package influence, together with their experimental validations.

\section{Acknowledgments}

The authors would like to thank Borka Lazarova for the execution of some experimental measurements.

In the end, the authors would like to thank the ECSEL JOINT UNDERTAK425 ING, in the international program H2020-ECSEL, G.A. nr. 826452, Arrowhead Tools project, for the partial funding of this work.

\section{References}

[1] G. Massimino, A. Colombo, L. D'Alessandro, F. Procopio, R. Ardito, M. Ferrera, A. Corigliano, Multiphysics modelling and experimental val- 
idation of an air-coupled array of PMUTs with residual stresses, Journal of Micromechanics and Microengineering 28 (5) (2018) 054005.

[2] Y. Lu, D. A. Horsley, Modeling, fabrication, and characterization of piezoelectric micromachined ultrasonic transducer arrays based on cavity SOI wafers, Journal of Microelectromechanical Systems 24 (4) (2015) 11421149. doi:10.1109/JMEMS . 2014.2387154.

[3] O. Rozen, S. T. Block, X. Mo, W. Bland, P. Hurst, J. M. Tsai, M. Daneman, R. Amirtharajah, D. A. Horsley, Monolithic MEMS-CMOS ultrasonic rangefinder based on dual-electrode PMUTs, in: Proceedings of the IEEE International Conference on Micro Electro Mechanical Systems (MEMS), 2016, pp. 115-118. doi:10.1109/MEMSYS.2016.7421571.

[4] X. Chen, J. Xu, H. Chen, H. Ding, J. Xie, High-accuracy ultrasonic rangefinders via PMUTs arrays using multi-frequency continuous waves, Journal of Microelectromechanical Systems 28 (4) (2019) 634-642. doi: 10.1109/JMEMS. 2019.2912869.

[5] X. Chen, J. Xu, H. Ding, X. Liu, D. Chen, J. Xie, A high-accuracy in-air reflective rangefinder via PMUTs arrays using multi-frequency continuous waves, 2019, pp. 154-157. doi:10.1109/TRANSDUCERS.2019.8808651.

[6] R. Ardito, E. Bertarelli, A. Corigliano, G. Gafforelli, On the application of piezolaminated composites to diaphragm micropumps, Composite Structures 99 (2013) 231-240. doi:10.1016/j.compstruct.2012.11.041

[7] S. Yazdi, A. Corigliano, R. Ardito, 3-D design and simulation of a piezoelectric micropump, Micromachines 10 (4). doi:10.3390/mi10040259

[8] K. M. Smyth, C. G. Sodini, S. Kim, High electromechanical coupling piezoelectric micro-machined ultrasonic transducer (PMUT) elements for medical imaging, in: TRANSDUCERS 2017 - 19th International Conference on Solid-State Sensors, Actuators and Microsystems, 2017, pp. 966-969. doi:10.1109/TRANSDUCERS. 2017.7994211. 
[9] Y. Lu, H. Tang, S. Fung, Q. Wang, J. M. Tsai, M. Daneman, B. E. Boser, D. Horsley, Ultrasonic fingerprint sensor using a piezoelectric micromachined ultrasonic transducer array integrated with complementary metal oxide semiconductor electronics, Applied Physics Letters 106 (26) (2015) 263503. doi:10.1063/1.4922915.

[10] G. Massimino, L. D'Alessandro, F. Procopio, R. Ardito, M. Ferrera, A. Corigliano, Air-coupled PMUT at $100 \mathrm{kHz}$ with PZT active layer and residual stresses: Multiphysics model and experimental validation, in: 18th International Conference on Thermal, Mechanical and Multi-Physics Simulation and Experiments in Microelectronics and Microsystems, EuroSimE 2017, 2017, p. 7926253. doi:10.1109/EuroSimE.2017.7926253.

[11] S. Trolier-McKinstry, P. Muralt, Thin film piezoelectrics for MEMS, Journal of Electroceramics 12 (2004) 7-17. doi:10.1023/B: JECR. 0000033998. 72845.51 .

[12] A. Corigliano, R. Ardito, C. Comi, A. Frangi, A. Ghisi, S. Mariani, Mechanics of Microsystems, John Wiley \& Sons, 2018.

[13] O. Rozen, S. T. Block, X. Mo, W. Bland, P. Hurst, J. M. Tsai, M. Daneman, R. Amirtharajah, D. A. Horsley, Monolithic MEMS-CMOS ultrasonic rangefinder based on dual-electrode PMUTs, in: 2016 IEEE 29th International Conference on Micro Electro Mechanical Systems (MEMS), IEEE, 2016, pp. 115-118. doi:10.1109/MEMSYS.2016.7421571.

[14] M. Amabili, Nonlinear vibrations and stability of shells and plates, Cambridge University Press, 2008. doi:https://doi.org/10.1017/ CB09780511619694.

[15] M. Ducceschi, C. Touzé, Modal approach for nonlinear vibrations of damped impacted plates: Application to sound synthesis of gongs and a cymbals, Journal of Sound and Vibration 344 (2015) 313-331. doi:https: //doi.org/10.1016/j.jsv.2015.01.029. 
[16] G. Massimino, A. Colombo, R. Ardito, F. Quaglia, F. Foncellino, A. Corigliano, Air-coupled array of PMUTs at $100 \mathrm{khz}$ with PZT active layer: Multiphysics model and experiments, in: 2019 20th International Conference on Thermal, Mechanical and Multi-Physics Simulation and Experiments in Microelectronics and Microsystems (EuroSimE), IEEE, 2019, pp. 1-5. doi:10.1109/EuroSimE.2019.8724514.

[17] W. Liu, L. He, X. Wang, J. Zhou, W. Xu, N. Smagin, M. Toubal, H. Yu, Y. Gu, J. Xu, D. Remiens, J. Ren, 3D fem analysis of high-frequency AlNbased PMUT arrays on cavity SOI, Sensors 19 (20) (2019) 4450. doi: $10.3390 / \mathrm{s} 19204450$

[18] L. E. Kinsler, A. R. Frey, A. B. Coppens, J. V. Sanders, Fundamentals of Acoustics, John Wiley \& Sons, 2000.

[19] G. Massimino, L. D'Alessandro, F. Procopio, R. Ardito, M. Ferrera, A. Corigliano, Multiphysics analysis and experimental validation of an air coupled piezoelectric micromachined ultrasonic transducer with residual stresses, Procedia Engineering 168 (2016) 852-855. doi:10.1016/j. proeng.2016.11.289

[20] M. Malinen, M. Lyly, P. Råback, A. Kärkkäinent, L. Kärkkäinen, A finite element method for the modeling of thermo-viscous effects in acoustics, in: ECCOMAS 2004 - European Congress on Computational Methods in Applied Sciences and Engineering, 2004.

[21] A. Frangi, G. Gobat, Reduced order modelling of the non-linear stiffness in MEMS resonators, International Journal of Non-Linear Mechanics 116

1. (2019) 211-218. doi:https://doi.org/10.1016/j.ijnonlinmec.2019. 07.002

[22] J. Chen, W. Ro, J. Lin, Exact static and dynamic critical loads of a sinusoidal arch under a point force at the midpoint, International Journal

口 of Non-Linear Mechanics 44 (1) (2009) 66-70. doi:https://doi.org/10. 1016/j.ijnonlinmec.2008.08.006 
[23] G. J. Simitses, Dynamic stability of suddenly loaded structures, Springer Science \& Business Media, 2012.

[24] A. H. Nayfeh, D. T. Mook, Nonlinear oscillations, John Wiley \& Sons, 2008.

[25] M. F. Hamilton, D. T. Blackstock, Nonlinear acoustics, Vol. 237, Academic

[26] M. I. Younis, MEMS linear and nonlinear statics and dynamics, Vol. 20, Springer Science \& Business Media, 2011.

[27] M. I. Younis, A. Nayfeh, A study of the nonlinear response of a resonant microbeam to an electric actuation, Nonlinear Dynamics 31 (1) (2003) 91-

[30] V. Zega, G. Langfelder, L. G. Falorni, C. Comi, Hardening, softening, and linear behavior of elastic beams in MEMS: An analytical approach, 535 ㄴ Journal of Microelectromechanical Systems 28 (2) (2019) 189-198. doi: 10.1109/JMEMS. 2019.2892296.

[31] M. I. Younis, H. M. Ouakad, F. M. Alsaleem, R. Miles, W. Cui, Nonlinear dynamics of MEMS arches under harmonic electrostatic actuation, Journal

匹 of Microelectromechanical Systems 19 (3) (2010) 647-656. doi:10.1109/ 
[32] K. K. Park, H. J. Lee, P. Crisman, M. Kupnik, O. Oralkan, B. T. KhuriYakub, Optimum design of circular CMUT membranes for high quality factor in air, in: Proceedings - IEEE Ultrasonics Symposium, 2008, pp. 504-507. doi:10.1109/ULTSYM.2008.0123.

${ }_{545}$ [33] M. K. Singha, R. Daripa, Nonlinear vibration and dynamic stability analysis of composite plates, Journal of Sound and Vibration 328 (4-5) (2009) 541-554. doi:https://doi.org/10.1016/j.jsv.2009.08.020.

[34] M. Rafiee, X. Q. He, K. M. Liew, Non-linear dynamic stability of piezoelectric functionally graded carbon nanotube-reinforced composite plates with initial geometric imperfection, International Journal of Non-

口 Linear Mechanics 59 (2014) 37-51. doi:https://doi.org/10.1016/j. ijnonlinmec.2013.10.011.

[35] H. Farokhi, M. H. Ghayesh, Nonlinear mechanics of electrically actuated microplates, International Journal of Engineering Science 123 (2018) 197213. doi:https://doi.org/10.1016/j.ijengsci.2017.08.017

[36] R. C. Batra, M. Porfiri, D. Spinello, Vibrations of narrow microbeams predeformed by an electric field, Journal of Sound and Vibration $309(3-5)$ (2008) 600-612. doi:https://doi.org/10.1016/j·jsv.2007.07.030.

[37] Q. Wang, S. Quek, C. Sun, X. Liu, Analysis of piezoelectric coupled circular plate, Smart Materials and Structures 10 (2) (2001) 229.

[38] O. Thomas, J.-F. Deü, J. Ducarne, Vibrations of an elastic structure with shunted piezoelectric patches: Efficient finite element formulation and electromechanical coupling coefficients, International Journal for Numerical Methods in Engineering 80 (2) (2009) 235-268. doi:10.1002/nme.2632.

565 [39] M. Sathyamoorthy, Nonlinear vibration analysis of plates: a review and survey of current development:doi:https://doi.org/10.1115/1.3149544.

[40] H. M. Ouakad, M. I. Younis, The dynamic behavior of MEMS arch resonators actuated electrically, International Journal of Non-Linear 
Mechanics 45 (7) (2010) 704-713. doi:https://doi.org/10.1016/j.

ijnonlinmec.2010.04.005. 